\title{
The Role of Cardiovascular Magnetic Resonance in Congenital Heart Disease
}

Authors: Rajesh Puranik, Vivek Muthurangu and Andrew MTaylor

Cardiovascular Magnetic Resonance Unit, Great Ormond St Hospital

Address for correspondence:

Dr Andrew MTaylor

Director of the Cardiovascular Magnetic Resonance Unit

Great Ormond St Hospital

Great Ormond St

London WC IN 3JH

Email:

a.taylor@ich.ucl.ac.uk

ABSTRACT The increasing prevalence of congenital heart disease (CHD) can be attributed to major improvements in diagnosis and treatment. Although echocardiography is the most commonly used imaging modality for diagnosis and follow up of subjects with CHD, the evolution of both cardiovascular magnetic resonance (MR) imaging and computed tomography (CT) does offer new ways to visualize the heart and the great vessels. The development of cardiovascular MR techniques such as spinecho and gradient-echo imaging, velocity-encoded phase contrast MR and gadolinium-enhanced MR angiography allow comprehensive assessment of cardiac anatomy and function. This provides information about the long-term sequelae of the underlying complex anatomy, hemodynamic assessment of residual post-operative lesions and complications of surgery. As much of the functional data in CHD patients is usually acquired with invasive $X$-ray angiography, non-invasive alternatives such as cardiovascular MR and CT are desirable. This review evaluates the role of both these modalities in the management of subjects with CHD, particularly detailing recent developments in imaging techniques as they relate to the various CHD diagnoses we commonly encounter in our practice.

\section{INTRODUCTION}

Major improvements in the diagnosis and treatment of congenital heart disease (CHD) have led to increased patient survival into adulthood. This has resulted in the greater prevalence of CHD in the general population. (I) A key component of the management of these patients is the requirement for life-long serial imaging. This allows investigation, and thus treatment, of the long-term sequelae of congenital heart disease, including late post-operative complications.

Echocardiography remains the most commonly used tool for diagnosis and follow-up of patients with CHD. The strengths of this modality include its ease of use, portability, and accuracy in assessing cardiac anatomy and function. However, echocardiography is limited by acoustic windows, provides poor images of distal vasculature and is highly operator-dependent. This is particularly the case in older children and adults, and in patients who have undergone multiple procedures. Therefore, cardiac catheterization remains an important component of both anatomic and functional assessment of patients with CHD. However, the clinical decision to proceed with such an investigation needs to be balanced with the risks associated with an invasive procedure, which also involves exposure to ionizing radiation. Furthermore, X-ray fluoroscopy provides a projection image, from which complex threedimensional (3D) morphologies can be difficult to discern.

Thus, the development of alternative imaging modalities is desirable in order to improve management of these patients. Both magnetic resonance (MR) imaging and computed tomography (CT) are becoming increasingly important in the diagnosis and management of $\mathrm{CHD}$ (Figure I). Such cross-sectional imaging techniques offer the ability to comprehensively assess cardiovascular anatomy and function. Further these newer modalities also provide an avenue to devise novel "hybrid therapeutic options" where imaging can not only guide but also directly assist in the treatment of congenital lesions.

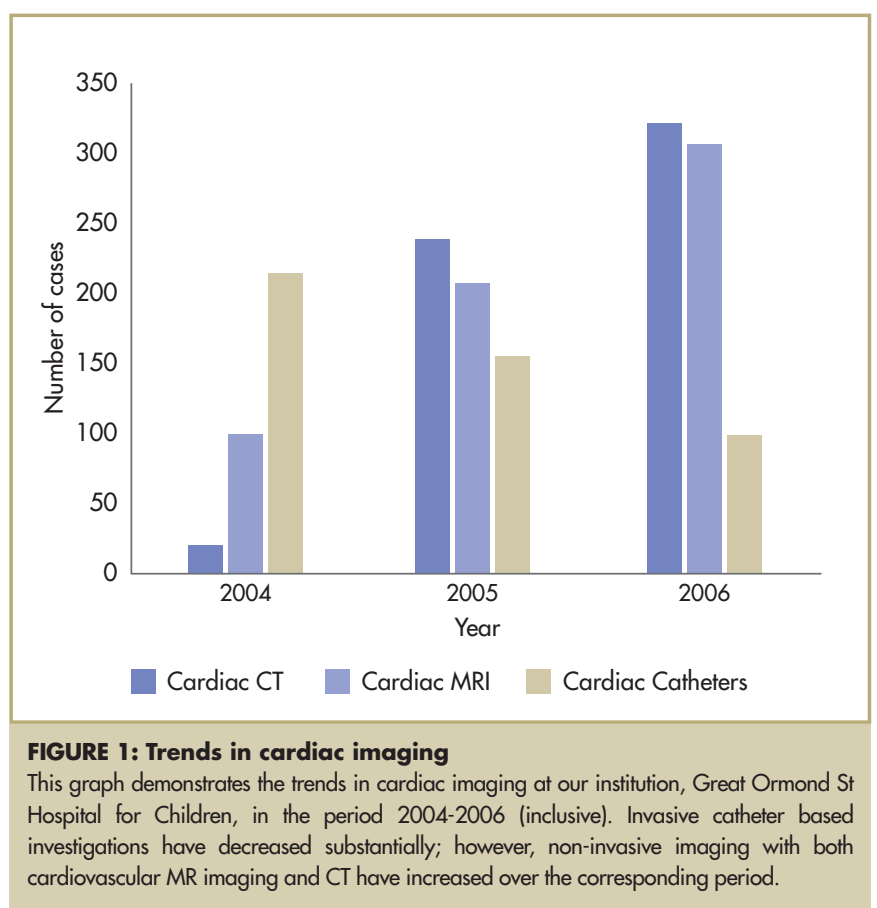


This review discusses the use of both MR and CT in the diagnosis and management of various CHD conditions. In particular, specific techniques that have evolved to optimize image quality and acquisition will be highlighted.

\section{MAGNETIC RESONANCE IMAGING}

MR imaging has been used for anatomical and functional evaluation of CHD for almost 20 years. ${ }^{(2-5)}$ Some of the advantages of cardiovascular MR include a large field of view, unlimited choice of imaging planes and low operator-dependency. In our practice, cardiovascular MR has an increasing role in the diagnosis and subsequent management of several forms of CHD (Figure I). In the section below we detail the most common forms of CHD that benefit from evaluation with crosssectional imaging. We will concentrate on aspects that are relevant to grown-up congenital heart disease ( $\mathrm{GUCH})$.

\section{Specific Imaging Techniques}

Spin echo "black blood" sequences were amongst the first used in cardiovascular MR imaging and they still remain an important part of current imaging strategies. ${ }^{(6)}$ In such sequences, flowing blood is black because it does not experience both the initial RF pulse and the refocusing pulse. Thus, contrast is improved with spin-echo black blood imaging at sites of accelerated blood flow velocity. Consequently, this technique is particularly useful for the assessment of sites of vascular obstruction and valvar stenosis. Black blood imaging is also useful when imaging vessels after metallic stent insertion, as the refocusing pulse compensates for increased T2* effects. This is useful as the majority of stents are safe to image within the MRI environment. ${ }^{(7)}$

Gradient echo-imaging has revolutionized cardiovascular MR as it allows dynamic imaging of cardiac anatomy. Gradient sequences have shorter repeat times and therefore have higher temporal resolution. The fast acquisition enables multiple phases of the cardiac cycle to be acquired, which can then be reconstructed into a cine MR image representing one full cardiac cycle. Single-slice two dimensional (2D) balanced steady-state free precession (b-SSFP) imaging allows qualitative assessment of all cardiac chambers, valvar dysfunction, and dynamic vascular anatomy. This form of imaging is particularly useful in planning interventions, as it allows accurate measurements of lesion size over the entire cardiac cycle. ${ }^{(8)}$ Multi-slice, 2D gradient echo imaging is currently regarded the best available in vivo method of assessing ventricular volumetry, ${ }^{(9)}$ as it is not reliant on complex geometric models.
Velocity-encoded phase-contrast MR enables accurate non-invasive blood flow quantification in major vessels. Cardiac output, pulmonaryto-systemic flow ratio and quantification of gradients across stenoses and valvar regurgitation can all be accurately determined using this technique. Volume blood flow measured using this technique has been shown to be accurate when compared to both invasive oximetry ${ }^{(10)}$ and flow-phantom experiments. ${ }^{(10)}$ In the case of stenoses interrogation, excellent comparative data exists with Doppler echocardiography and catheter data. (II) The quantification of regurgitant volume using this technique has been demonstrated to be superior to echocardiography. ${ }^{(12)}$

Gadolinium-enhanced MR angiography (Gd-MRA) relies on the TIshortening effect of dilute gadolinium and it has been shown to be useful in delineating thoracic vascular anatomy. Gadolinium imaging allows volume scans to be obtained within a breath hold, thus reducing respiratory artifact. Gd-MRA is useful in identifying stenotic vessels and correlates well with modalities such as X-ray angiography and surgical findings. ${ }^{(13)}$ It should be noted MRA imaging is not ECG-gated and thus dimensions measured from these images represent an average of dimensions throughout the cardiac cycle.

\section{Tetralogy of Fallot}

Tetralogy of Fallot (ToF) (Figure 2) is the most common cyanotic congenital heart defect, with an incidence of approximately 420 per million live births. ${ }^{(14)}$ Mal-alignment of the septum leads to right ventricular outflow tract (RVOT) obstruction, a sub-aortic ventricular septal defect (VSD) with aortic override, and right ventricular hypertrophy. Current management consists of early single-stage reconstructive surgery, ${ }^{(15)}$ with staged reconstruction now reserved for cases with significant hypoplasia of the central pulmonary arteries. ${ }^{(16)}$ Gd-MRA of the pulmonary arteries correlates well with operative and conventional X-ray angiographic findings and may be useful in determining the most appropriate corrective treatment strategy. ${ }^{(1,17)}$

However, the main role of MR in patients with ToF is assessment of postoperative complications. Operative repair of ToF consists of resection of the infundibular stenosis, enlargement of the pulmonary valve/annulus and closure of the VSD. Pulmonary regurgitation due to previous RVOT/valvar surgery with transannular patch reconstruction of the RVOT/pulmonary annulus is the most common late postoperative complication. ${ }^{(18,19)}$ Current strategies for the management of severe pulmonary regurgitation include surgical and trans-catheter approaches, which rely on accurate quantification of regurgitation and definition of the RVOT anatomy. . $^{(20)}$ 


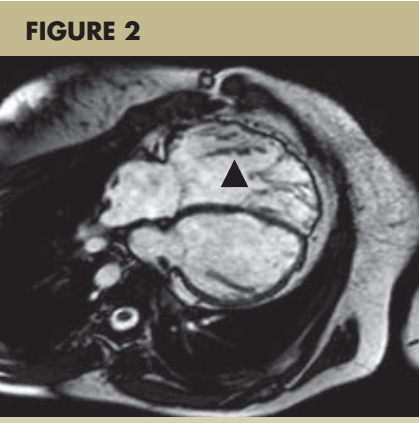

A: Balanced-SSFP four-chamber image showing RV dilatation (triangle) in a patient with Tetralogy of Fallot (ToF).

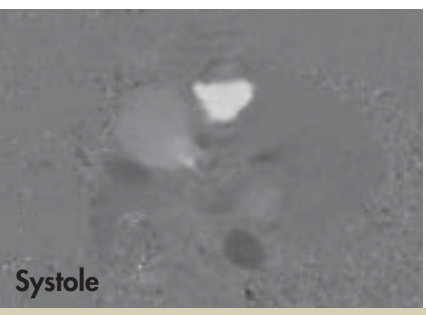

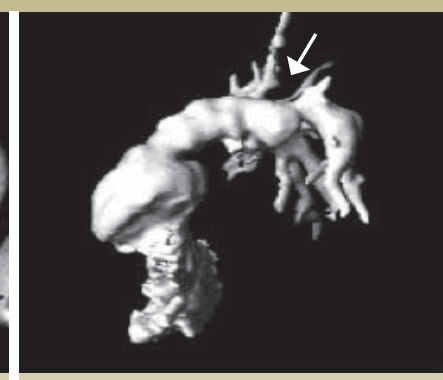

B: Volume-rendered 3D reconstruction from gadolinium-enhanced MR angiogram demonstrating pulmonary artery stenosis (arrow) in a patient with ToF.

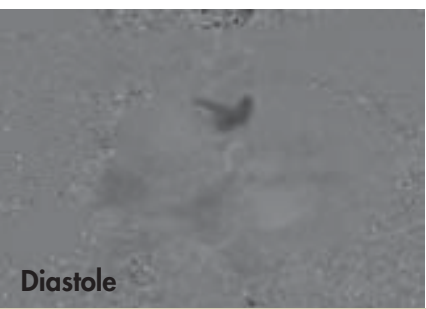

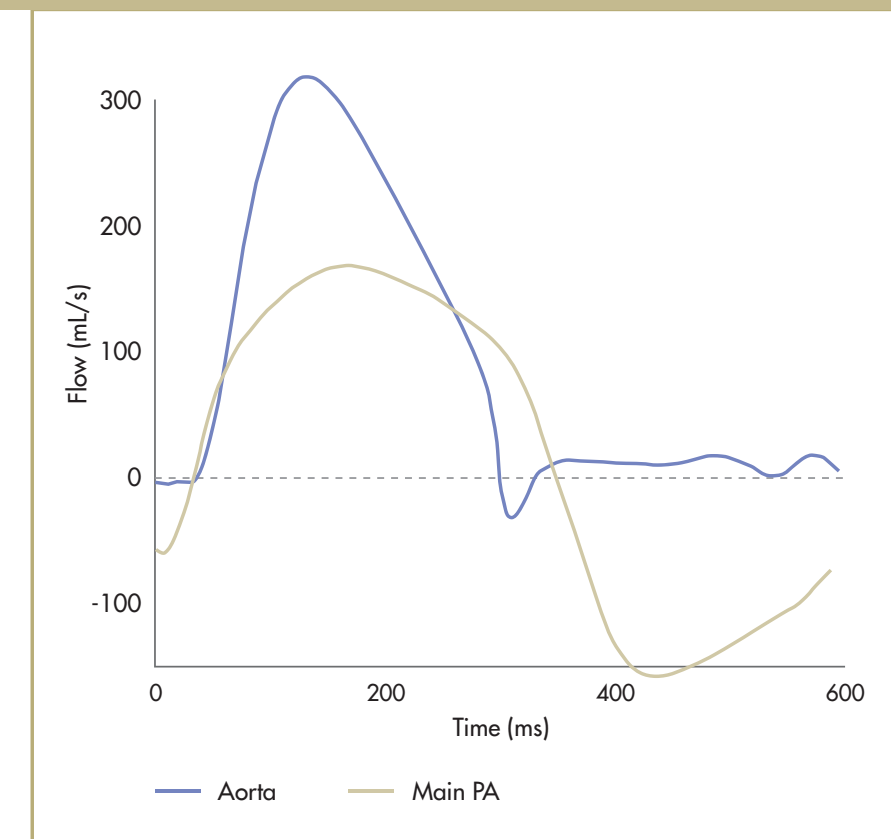

C: Magnetic resonance phase-contrast flow images through the main pulmonary artery (PA): Forward flow in systole (shown as white) and diastolic flow (shown as black) are depicted above. Flow is then plotted against time for the aorta and main PA, demonstrating pulmonary incompetence (regurgitant fraction=30\%) in the lower panel.

2D spin-echo black blood and b-SSFP sequences can be used to define RVOT anatomy and quantitatively assess RVOT dilatation or stenosis. Velocity-encoded phase-contrast MR can accurately quantify the degree of pulmonary regurgitation and has been internally validated against MR ventricular volumetry in patients with ToF.(12) In addition, velocity-encoded phase contrast MR can be used to measure peak velocities at the level of RVOT obstruction, and differential regurgitation in the branch pulmonary arteries. ${ }^{(21)}$ These parameters have excellent correlation with findings from echocardiography and catheter data. ${ }^{(11,22)}$

MR assessment of right ventricular function is particularly important when determining the timing and evaluating the impact of invasive therapeutic strategies. ${ }^{(8,23)}$ Multi-slice short-axis b-SSFP imaging has been shown to be the most powerful method of quantifying ventricular function. ${ }^{(9)}$ Furthermore, a combination of MR ventricular volumetry and tricuspid and pulmonary flow maps can be used to provide precise information about global and diastolic ventricular function. ${ }^{(24)}$

Pulmonary atresia, regarded as an extreme variant of ToF, is defined as a lack of continuity between the RVOT and the central pulmonary arteries, with a variable degree of hypoplasia of these structures. MR has a similar pre- and post-operative role in subjects with pulmonary atresia and aVSD as it does in ToF patients. Gd-MRA allows assessment of the central pulmonary arteries and major aorto-pulmonary collateral arteries (MAPCAs). ${ }^{(25)}$ 3D visualization enhances surgical or interventional planning beyond the data provided by $\mathrm{X}$-ray angiography. The post-operative functional quantification of homograft regurgitation and stenosis is performed with velocity-encoded phase contrast MR, while black-blood imaging can be used to demonstrate stenosed conduits. ${ }^{(26)}$ However, it should be noted that under-reporting of conduit stenosis due to calcification is possible with this technique.(26)

\section{Transposition of the Great Arteries}

Transposition of the great arteries (TGA) (Figure 3) is the secondcommonest cyanotic CHD diagnosed in the first year of life, with an incidence of 315 per million live births. ${ }^{(14)}$ Currently, echocardiography is the imaging modality of choice for pre-operative diagnosis and assessment. As in ToF, the main role of MR is in diagnosis of postoperative complications, particularly those that develop as the child grows older.

Treatment for TGA patients was revolutionized with the introduction of the Senning procedure, in which an intra-atrial baffle was used to divert blood from the right atrium to the left ventricle, and the left atrium to the right ventricle. A variation to the Senning procedure, the Mustard procedure uses a pericardial patch to construct the intra-atrial baffle. However, although both these procedures produce a physiologically normal circulation, the patient is still left with a systemic right ventricle. Therefore, the arterial switch operation has become the 
procedure of choice. In cases of TGA associated with a VSD and subpulmonary stenosis, the Rastelli procedure is preferred.

Cardiovascular MR is not constrained by the intra-thoracic position of vessels and is ideal for imaging the RVOT and branch pulmonary arteries, the sites commonly where complications after the arterial switch procedure are identified. ${ }^{27,28)}$ Gd-MRA is used to visualize the 3D anatomy, particularly the relationship between the pulmonary arteries and the aorta, while spin-echo sequences are used to accurately assess sites of stenoses. It has been shown that this combination of techniques is superior to echocardiography and compares favorably with conventional $X$-ray angiography in detecting significant lesions. ${ }^{(29)}$ Velocity-encoded phase contrast MR can be used to measure peak velocities at the level of the obstruction and calculate pressure gradients. This data has been shown to have a greater agreement with invasive pressure measurements than corresponding pressure gradients derived from Doppler ultrasound. ${ }^{(28)}$ A less common complication of the arterial switch procedure is coronary stenosis secondary to the reimplantation procedure. Although $\mathrm{X}$-ray angiography represents the modality of choice, coronary MR angiography is particularly useful when assessing the proximal coronary arteries. ${ }^{(13)}$

Despite intra-atrial repair being superseded by the arterial switch operation, there are a significant number of patients who have undergone either a Senning or Mustard operation. As the venous pathways have a complex 3D structure they are difficult to accurately assess with echocardiography. Gd-MRA can completely demonstrate the 3D Senning-Mustard anatomy and detect any luminal narrowing. ${ }^{(30)}$ Spin-echo sequences can accurately measure static baffle obstruction, (31) while 2D and 3D b-SSFP sequences can delineate dynamic anatomy of intra-atrial baffles. ${ }^{(31)}$

\section{Aortic pathologies}

Coarctation accounts for 6-8\% of live births with CHD and refers to an area of narrowing of the thoracic aorta in the region of insertion of the arterial duct with or without additional abnormalities of the aortic arch. (32) Detailed serial follow up is mandatory as recoarctation occurs in $3-35 \%$ of surgically treated patients. ${ }^{(33)}$ Added to this is the fact that a third of patients remain or become hypertensive as well as having an increased lifetime risk of atherosclerosis and end-organ damage. Although angiography has been regarded as the "gold standard" for the assessment of coarctation, MR imaging has been shown to be diagnostic and well suited for both pre- and post-operative evaluation. ${ }^{(34,35)}$

Spin-echo imaging in the sagittal and oblique-sagittal planes provides excellent information of stenotic areas and collateral pathways. ${ }^{(36)}$ 3D CE-MRA can display the severity and extent of involvement without spin dephasing artifacts and partial volume errors. ${ }^{(37)}$ The severity of the stenosis at the level of the coarctation can be accurately determined using MR flow mapping. ${ }^{(38,39)}$

CE-MRA can be used to perform 3D post-processing techniques, which allow abnormalities to be viewed from different orientations. It should be emphasized that 3D MRA should be combined with at least one other MR sequence, usually black-blood or 3D b-SSFP imaging in

\section{FIGURE 3}
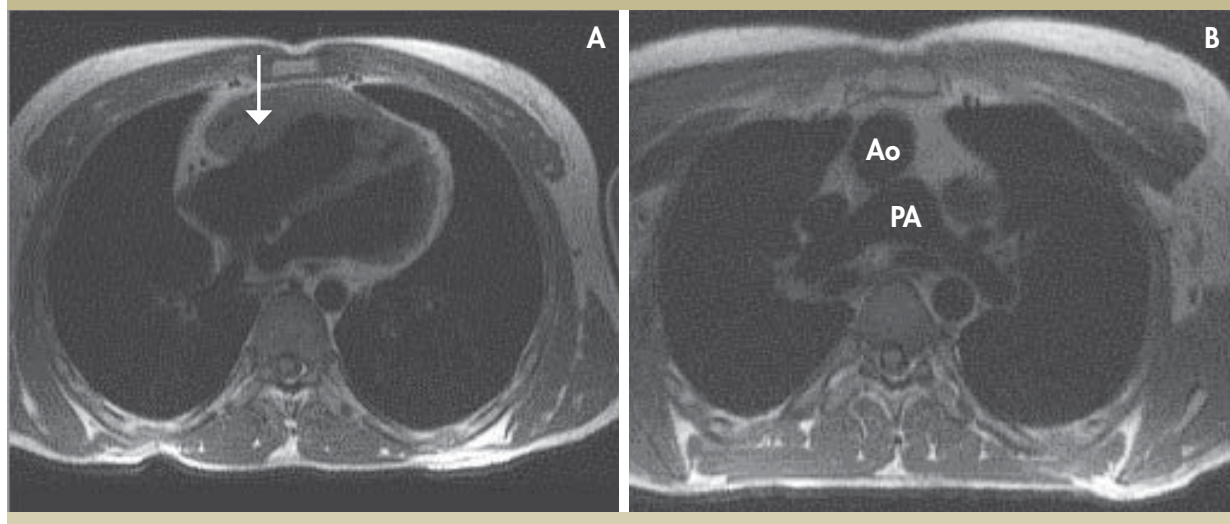

Axial black-blood turbo-spin-echo imaging (A, B) and b-SSFP sagittal imaging (C) of transposition of the great arteries. The aorta $(\mathrm{Ao})$ arises anteriorly from the hypertrophied right ventricle (arrow). The central pulmonary artery (PA) arises from the left ventricle. 
FIGURE 4: MRI of aortic coarctation. Images from various subjects.

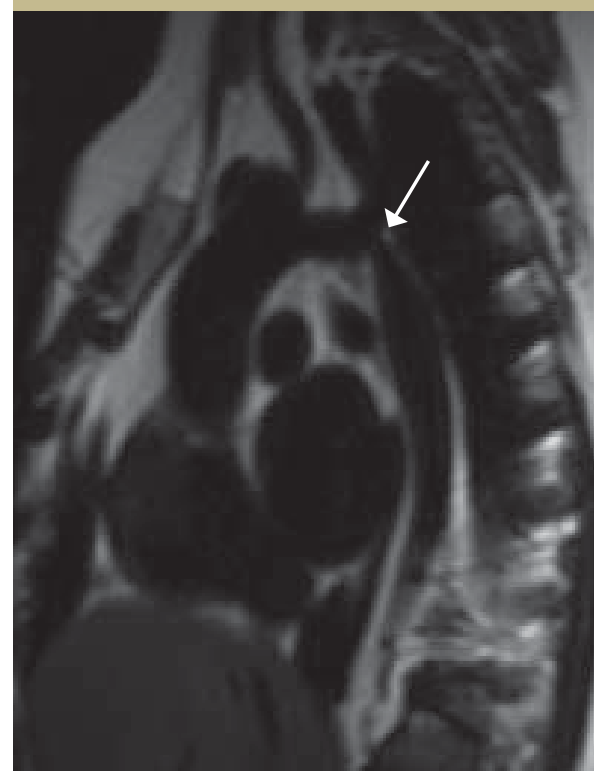

A: Black-blood turbo-spin-echo oblique sagittal image through the aorta showing a tight discrete aortic coarctation at the aortic isthmus (arrow).

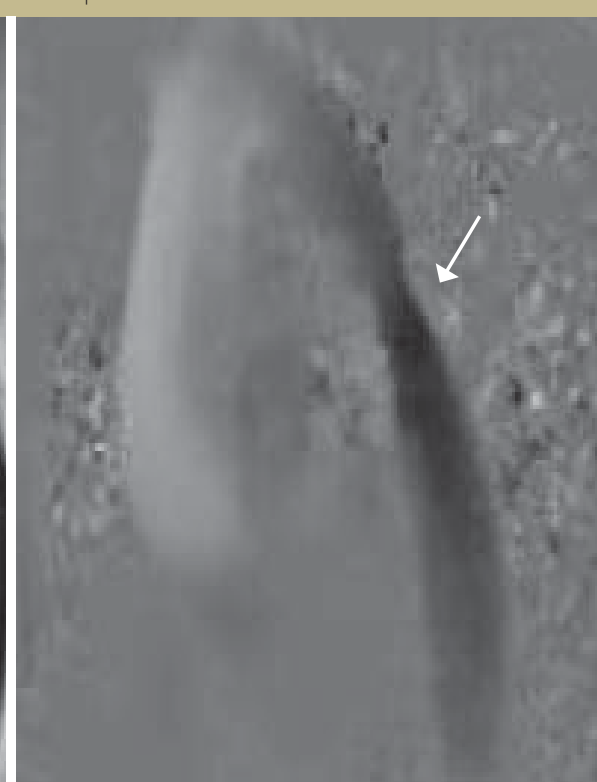

B: Velocity map images of a more distal moderate coarctation (arrow).

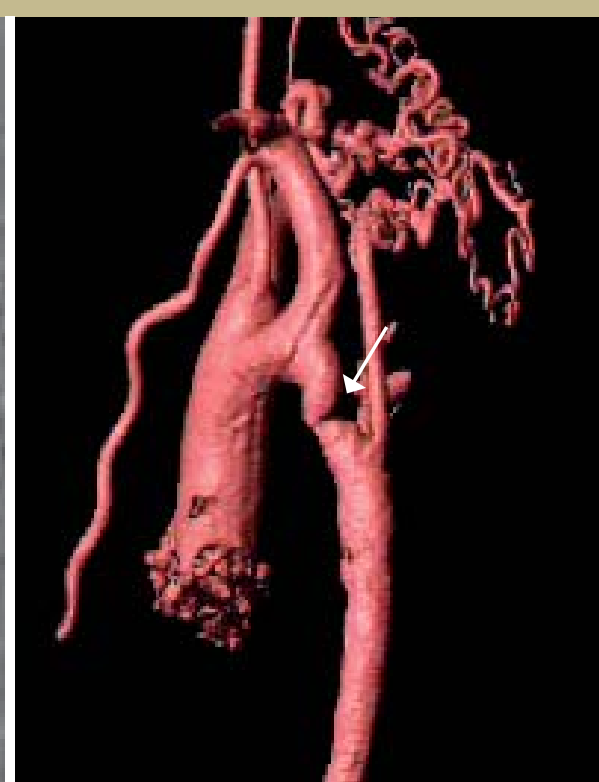

C: Volume-rendered 3D reconstruction of contrastenhanced MR angiography data in a third subject, showing severe coarctation (arrow) and multiple collateral vessels. the axial and sagittal oblique plane, in order to ensure that complete assessment of the aortic pathology is performed (Figure 4).

MR imaging should also be used to identify other secondary pathologies in these patients, including: assessment for the presence of dilatation of the aorta: aortic valve stenosis and regurgitation; and quantification of ventricular function and mass. MR follow-up after surgery or balloon dilatation allows accurate evaluation of recoarctation, residual stenosis, persistence of aortic arch hypoplasia and false aneurysm at the level of repair. ${ }^{(40,41)}$ Cine imaging of the heart should always accompany these studies, for detection of associated intra-cardiac lesions. ${ }^{(42)}$

Aortic dilatation and/or dissection are critical to both the diagnosis and prognosis of subjects with Marfan syndrome. MRI provides definitive measurements of aortic dimensions, which are essential for the diagnosis of aortic involvement and serial follow up measurements. ${ }^{(43)}$ Dilatation usually commences at the sinuses of Valsalva and MR derived diameters are particularly important in monitoring the early stages of this disease. Diameters must, however, be related to normal values for age and body surface area. ${ }^{(44,45)}$ Added to this, MR also enables the assessment of aortic biophysical properties, such as decreased aortic distensibility and increased flow wave velocity, in the aorta distal to the aortic root. These parameters have been shown to be present prior to overt aortic dilatation in subjects with Marfan syndrome (Figure 5).
Finally, there are an increasing number of patients being treated with aortic stents for both coarctation ${ }^{(46)}$ and aneurysms. ${ }^{(47)}$ Although these metallic implants are safe to image under MR, gradient echo sequences are prone to field inhomogeneity effects (T2*) related to the presence of the stent. Black-blood imaging provides the solution to this, with T2* effects compensated for by the $180^{\circ}$ refocusing pulse. Thus, patients with

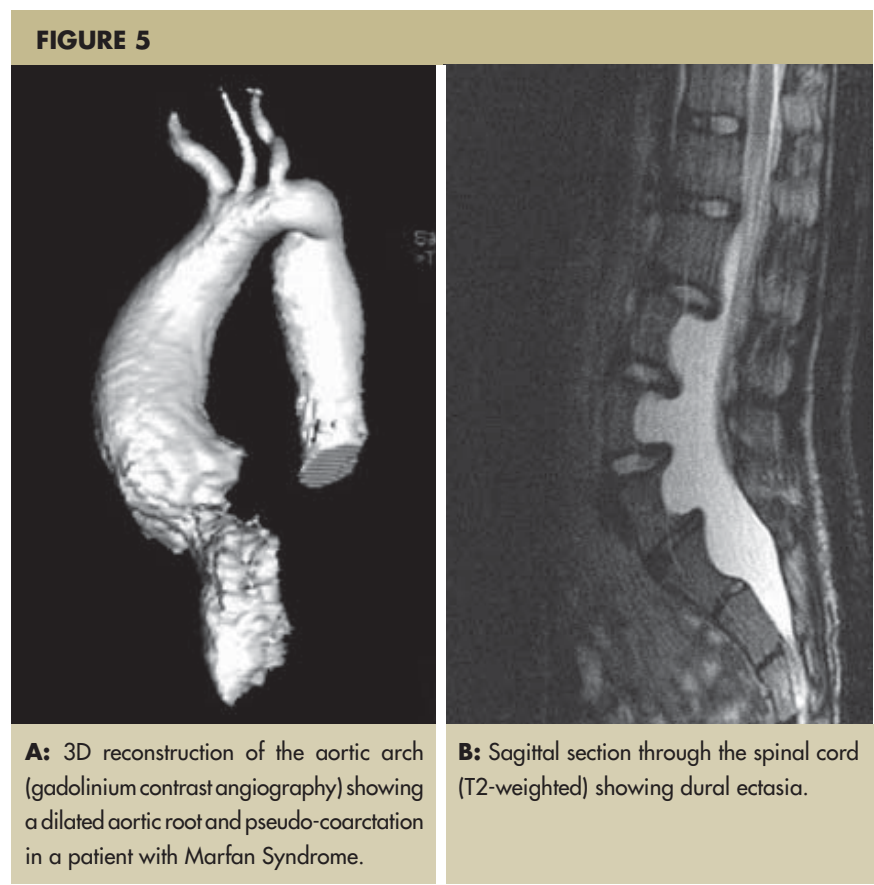


FIGURE 6: Aortic coarctation stent imaging

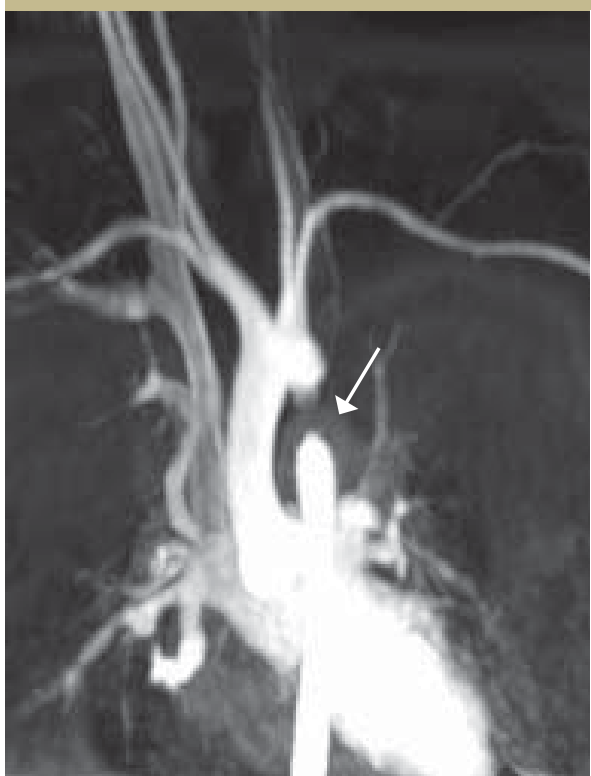

A: Coronal images from an aortic CE-MRA showing signal loss at the site of a proximal descending aorta metallic stent (arrow).

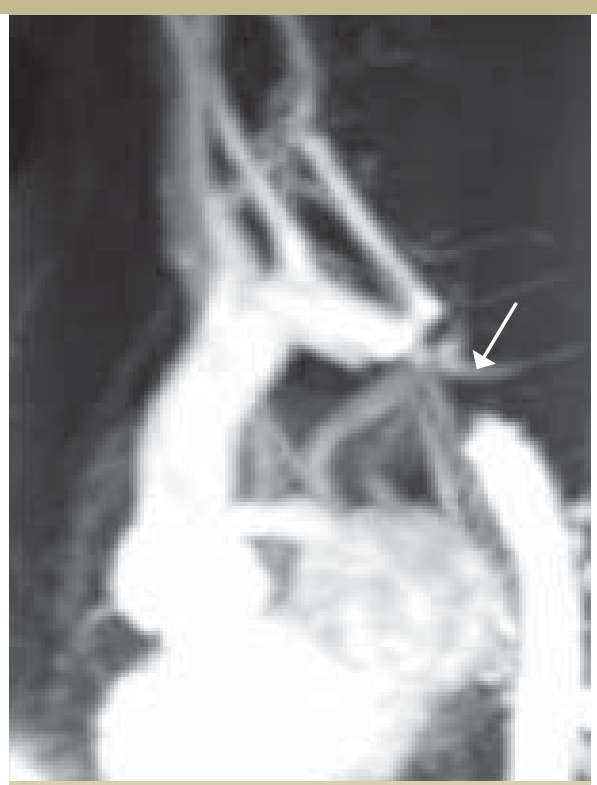

B: Sagittal images from an aortic CE-MRA showing signal loss at the site of a proximal descending aorta metallic stent (arrow).

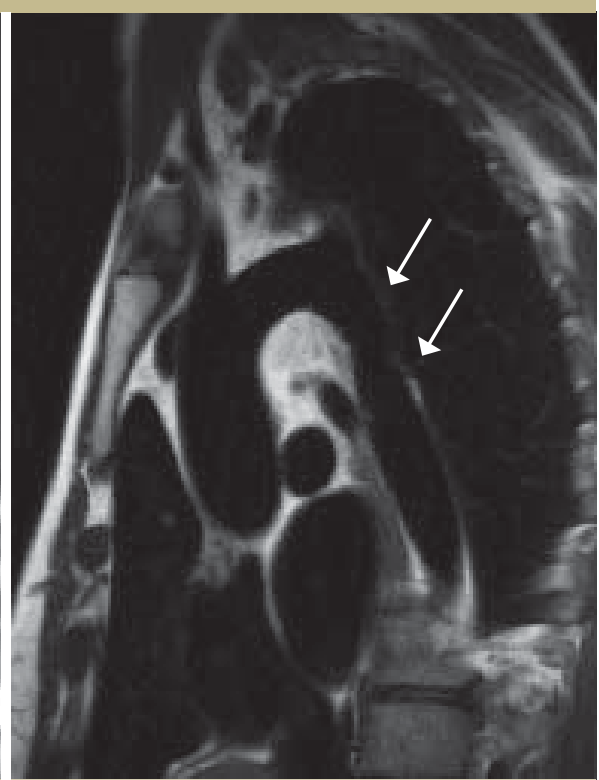

C: Oblique sagittal TSE black-blood image in the same patient clearly demonstrates that the stent is widely patent. The length of the stent is indicated by the arrowheads.

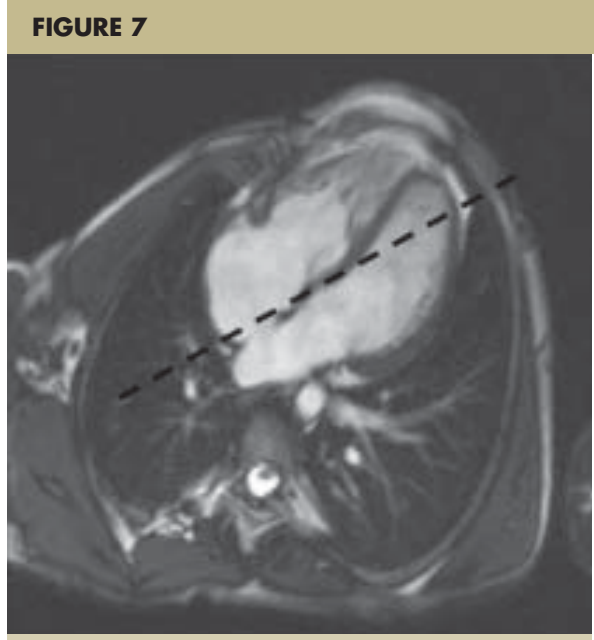

A: b-SSFP image through a secundum atrial septal defect. Note there is no posterior rim; precluding insertion of an ASD closure device.

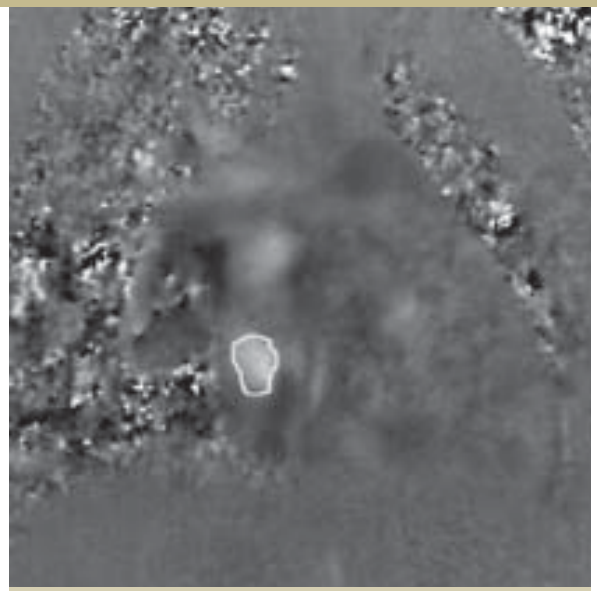

B: The dotted line is the plane for through-plane phaseencode velocity flow mapping.

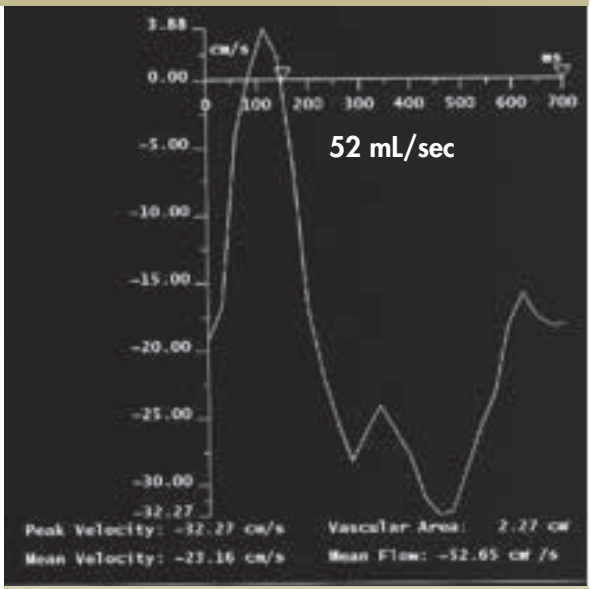

C: Instantaneous flow-against-time curve, through the ASD. The total flow through the ASD is $52 \mathrm{ml} /$ beat in a left to right direction. stents in-situ can still be assessed by MR, and restenosis, stent placement, stent and aortic wall morphology can be assessed ${ }^{(48)}$ (Figure 6).

\section{Atrial Septal Defect}

Atrial septal defects (ASD) (Figure 7) are the most common congenital heart defects detected in adults, with an incidence of 94I per million live births. ${ }^{(14)}$ The presence of an ASD allows left-to-right shunting, leading to atrial and ventricular dilatation, which can then predispose to arrhythmia and volume overload. ASDs are also an independent risk factor for thrombo-embolic stroke. Previously, surgical closure of ASDs was performed when there was the existence of a large left-to-right shunt, which led to RV overload, atrial dilatation and symptoms. The advent of transcatheter ASD closure techniques has resulted in a more aggressive management strategy. ${ }^{(49,50)}$ Small- to medium-sized ostium secundum defects are best suited to this approach, as a deficiency of the anterior or postero-inferior rim of the defect precludes transcatheter closure. 
Cardiovascular MR plays an important role in the diagnosis and preinterventional assessment of ASDs. In fact, MR findings have been shown to be more powerful predictors of successful catheter closure than transoesphageal or transthoracic echo findings. ${ }^{(51)}$ Two-dimensional (2D) spin-echo black-blood sequences and gradient-echo sequences accurately defines ASD anatomy ${ }^{(52,53)}$ and correlates well with findings at surgery. ${ }^{(54)}$ Multi-slice 2D gradient-echo techniques can be used to assess dynamic 3D anatomy of the defect. Multi-planar reformatting of this data can produce image planes that are useful in planning interventional procedures ${ }^{(55)}$ However, as multi-slice techniques suffer from poor through-plane resolution, 3D b-SSFP sequences with isotropic resolution have been developed to produce accurate multiplane reformatted images with no loss of resolution. ${ }^{(56)}$

Pulmonary venous abnormalities, which often accompany sinus venous defects, are well defined on Gd-MRA ${ }^{(57)}$ and this information can assist in surgical planning. Also, evaluation of the hemodynamic status of ASDs can be performed with cardiovascular MR. Quantification of left-toright shunts using velocity-encoded phase-contrast MR compares well with invasive catheterization derived data. ${ }^{(10)}$ Multi-slice SSFP short-axis imaging accurately quantifies the degree of ventricular overload, information that is critical in determining the timing of invasive intervention. ${ }^{(9,58)}$

\section{Ventricular Septal Defect}

VSDs are the most common CHD defects, with an incidence of 3,570 per million live births. ${ }^{(14)}$ Cardiovascular MRI has the advantage of offering a non-invasive method of quantifying left-to-right shunts caused by VSDs. Velocity-encoded phase-contrast MR compares well with traditional invasive catheterization methods. ${ }^{(10)}$ Added to this there is preliminary evidence to suggest that in certain situations combining phase-contrast MR and invasive pressure measurements is more accurate than traditional methods alone, which rely on the Fick principle. ${ }^{(59)}$

Cardiovascular MR provides accurate 2D and 3D images of the defect. Multi-slice 2D gradient-echo techniques can be used to assess the dynamic 3D anatomy of the defect. In addition, multi-planar reformatting of this data can be used to produce image planes that are useful in planning interventional procedures. ${ }^{(55)}$ For example, the relationship of the VSD to valvar structures is an important consideration when deciding on transcatheter closure. Also, where there are multiple VSDs, palliative pulmonary artery banding adequacy ${ }^{(60)}$ can be accurately determined using velocity encoded gradient-echo cine MR and has been shown to compare well with surgical and catheter findings.

\section{Anomalous Coronary Arteries}

Anomalous coronary artery origin and proximal course is a recognized cause of myocardial ischemia and sudden death. ${ }^{(61)}$ Although X-ray angiography is regarded as the gold standard for evaluation of anomalous coronary arteries, findings from MR angiography correlate well and eliminate exposure to X-ray radiation. (62) Additionally, cardiovascular MR can also be used to assess perfusion and infarct size, ${ }^{(63)}$ which are powerful predictors of prognosis.

\section{MR IMAGING PROTOCOL FOR CHD}

The protocol below is a general protocol for imaging of CHD. It should be altered to answer clinical questions depending on patient anatomy.

I. Scouts scans

2. HASTE black-blood axial imaging

3. Navigator echo gated True FISP 3D volume of whole heart and great vessels

4. 2D single-slice True FISP cine images

a. Vertical long axis

b. Four-chamber view

c. RVOT in two planes

d. LVOT in two planes

e. Both branch pulmonary arteries

5. 2D multi-slice True FISP cine images - entirety of both ventricle in short-axis

6. 2D single-slice black-blood TSE imaging

a. Branch pulmonary arteries

b. Aortic arch

c. Vascular stenoses

7. Phase-contrast velocity mapping

a. Main pulmonary artery through-plane

b. Ascending aorta through-plane

c. Vascular stenoses in-plane

8. 3D contrast-enhanced MR angiography of the thoracic great vessels 


\section{THE ROLE OF COMPUTED TOMOGRAPHY IN CHD}

As discussed in the previous section, MR of the heart and great vessels is often used in the assessment of CHD, both in grown-up cases and more recently in neonates, infants and younger children. ${ }^{(33)}$ However with its increasing availability and utility, multi-detector computed tomography (MDCT) imaging is now becoming established as a further method of imaging CHD. The development of spiral(64,65) and subsequently $\operatorname{MDCT}^{(66,67)}$ enables the acquisition of data during a single breath-hold and during the first pass of a contrast bolus, so that images can be reconstructed in any two-dimensional plane or in three dimensions.

In our own practice, we currently use MDCT for the following indications in patients with CHD:

- Aortic pathology, in particular aortic arch vascular rings (+airways imaging) (Figure 8)

- Pulmonary artery anatomy (only if no functional information is required)

- Post-operative systemic to pulmonary shunts - size and patency

- Pulmonary venous anatomy - total and partial anomalous pulmonary venous drainage(64) (Figure 9)

\section{FIGURE 8}

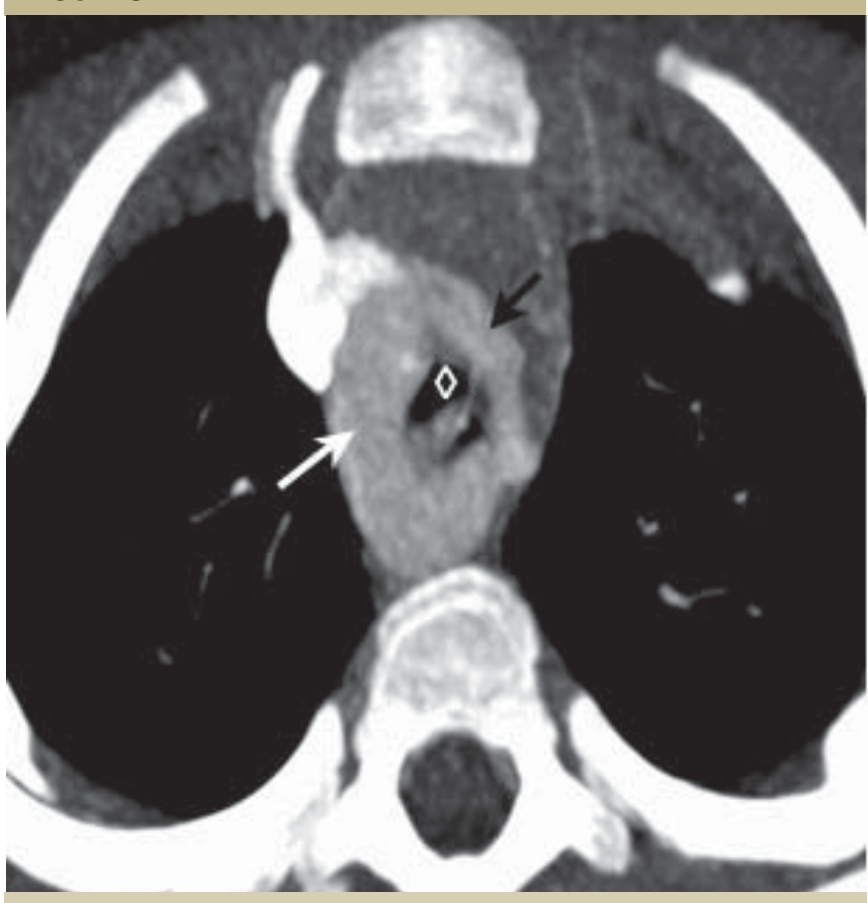

$\mathrm{CT}$ image demonstrating tracheal compression from a complete vascular ring - double aortic arch. The oesophagus (diamond), right aortic arch (white arrow) and left aortic arch (black arrow) are shown.

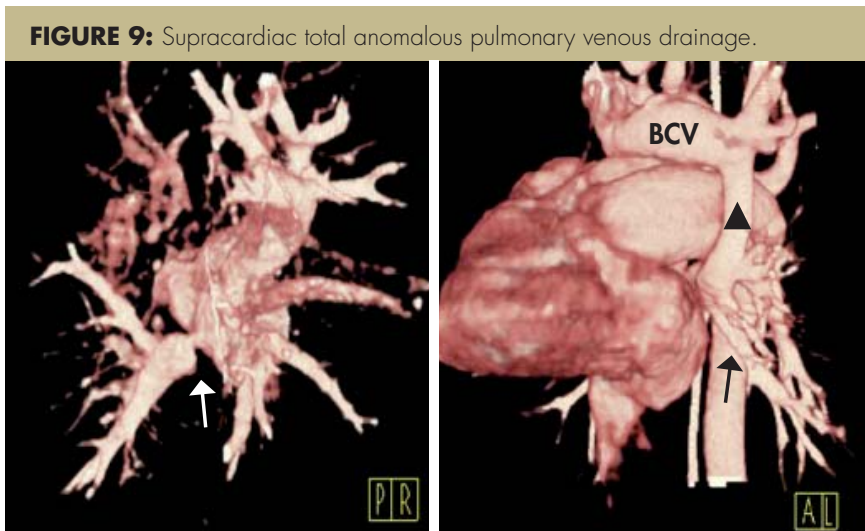

A: 3D volume-rendered $M D C T$ angiogram viewed from left anterior oblique showing a supracardiac total anomalous pulmonary venous drainage.

B: The pulmonary veins (arrow) drain into an ascending vein (triangle) and then into the brachiocephalic vein ("BCV") and SVC which drains into the right atrium.

Vascular stent imaging (Figure 10)

- Contraindication to MR imaging - permanent pacemaker, cerebral aneurysm clips, etc.

\section{MR Versus MDCT}

A major advantage of the recent advances in CT technology (16-slice and 64-slice MDCT) over MR is the very rapid acquisition time. Imaging can now be performed in a comfortable single breath hold at the peak of the contrast bolus, reducing the need for general anesthesia and sedation in young children. Currently, it is our policy to perform MR imaging in children less than 7 years of age under general anesthesia. Thus, in this age group, when anatomical information alone is needed, we will perform a MDCT scan (unsedated or sedated). However, if quantification of ventricular function or vascular flow (e.g. pulmonary incompetence) is required we will perform an MR scan under general anesthesia. For subjects older than 7 years of age, and no contraindications, we will perform MR imaging in the first instance. If there are contraindications to MR imaging (permanent pacemaker, cerebral aneurysm clips, etc.) or when stent imaging is necessary (metal artifact on MR imaging), we will perform cardiovascular MDCT.

There are two main disadvantages of MDCT compared with MR imaging. The first is the use of ionizing radiation. This can be kept to a minimum, by using low $\mathrm{kV}$, low mAs acquisitions, with current modulation, and image acquisition over the minimal area of interest. Using such protocols, our mean dose for non-cardiac gated cardiovascular MDCT in children is $1.2 \pm 0.57 \mathrm{mSv}$. This equates to approximately 60 chest radiographs (standard PA chest radiograph $=$ $0.02 \mathrm{mSv}$ ) or 6 months of background radiation exposure (UK average background radiation $=2.2 \mathrm{mSv}$ per year). ${ }^{(68,69)}$ Furthermore, in critically ill patients the ability to perform a rapid examination without the need 
for general anesthesia or even sedation may be more crucial, as the risk of prolonged sedation may be greater than that of radiation. ${ }^{(67)}$ The second disadvantage of current MDCT techniques is that easy quantification of cardiac function (at high heart rates) and arterial flow are not possible, especially when compared with cardiac-gated MR imaging.

Importantly, as our GUCH population ages, patients will become more prone to ischemic heart disease. Over the next 5 years, MDCT will become increasingly used to assess the coronary arteries, and will probably be the best method to non-invasively investigate chest pain in this patient population. ${ }^{(70-72)}$

\section{Future prospects}

MDCT scanning will undoubtedly become more rapid, with installation of 64-slice scanners, now beginning, and the development of 256-slice scanners in the near future. This will enable even faster data acquisition, improving spatial and temporal resolution. This should allow ECG-gated images of cardiac anatomy to be feasible even at high heart rates. The assessment of cardiac function will then be routinely possible. Furthermore, such equipment will facilitate accurate non-invasive assessment of coronary artery disease.

\section{CONCLUSION}

The recent improvements in non-invasive cross-sectional cardiovascular imaging modalities (MR and $\mathrm{CT}$ ) have resulted in a change in our approach to the assessment and follow up of patients with CHD. The precise role of these imaging modalities will evolve over time. Currently, our own clinical practice is to use echocardiography in all cases of CHD. However, echocardiography can be technically difficult to perform, providing sub-optimal imaging. In these situations we use cardiovascular MR to further define CHD anatomy and physiology. This is particularly important prior to and following corrective surgical and interventional procedures. Cardiovascular MR is critical to the non-invasive assessment of ventricular/valvar function and blood flow through hemodynamically significant lesions and shunts. We specifically use MDCT in the initial diagnostic assessment of great vessel anatomy in young subjects, especially in circumstances where functional information is not required. Finally we use cardiac catheterization/angiography if haemodynamic information is required (pulmonary vascular resistance studies), or if there is a high degree of suspicion of coronary artery stenoses. This approach can improve non-invasive diagnosis and reveal detailed anatomy that is important for both clinical decision-making and surgical planning.

\section{ACKNOWLEDGEMENTS}

RP is the Neil Hamilton Fairley NHMRC/NHF of Australia Overseas Post-doctoral Fellow. VM is funded by a grant from UCL Institute of Child Health. AMT is funded by the Higher Education Funding Council of England (HEFCE).

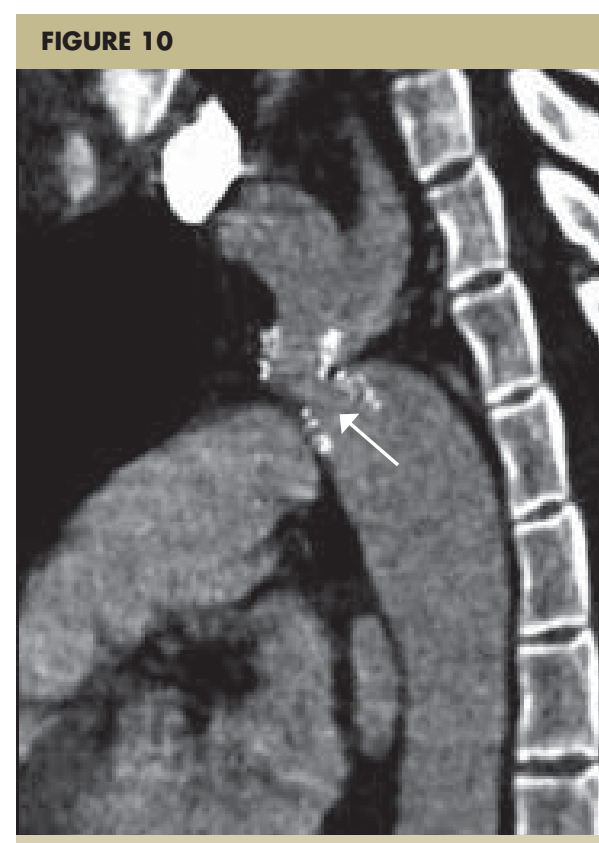

A: MDCT demonstrating stent fracture at the site of aortic coarctation.

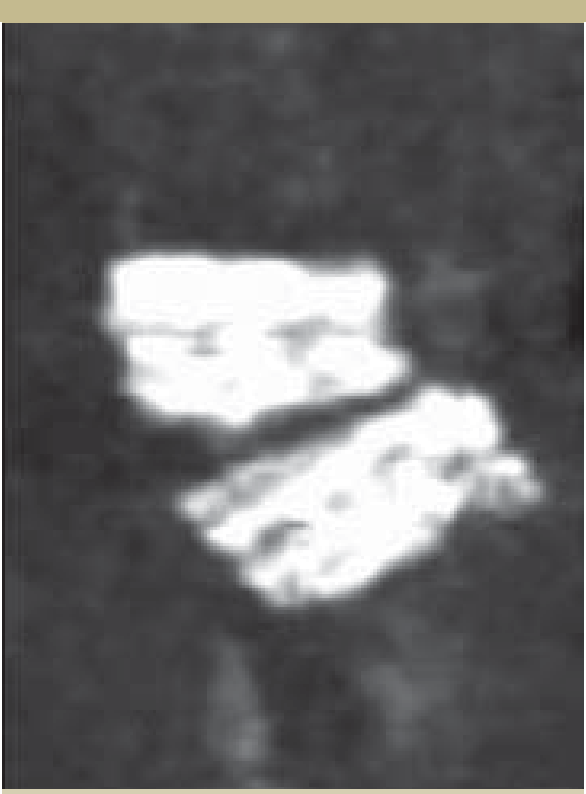

B: Aortic stent fracture shown at high magnification derived from MDCT imaging.

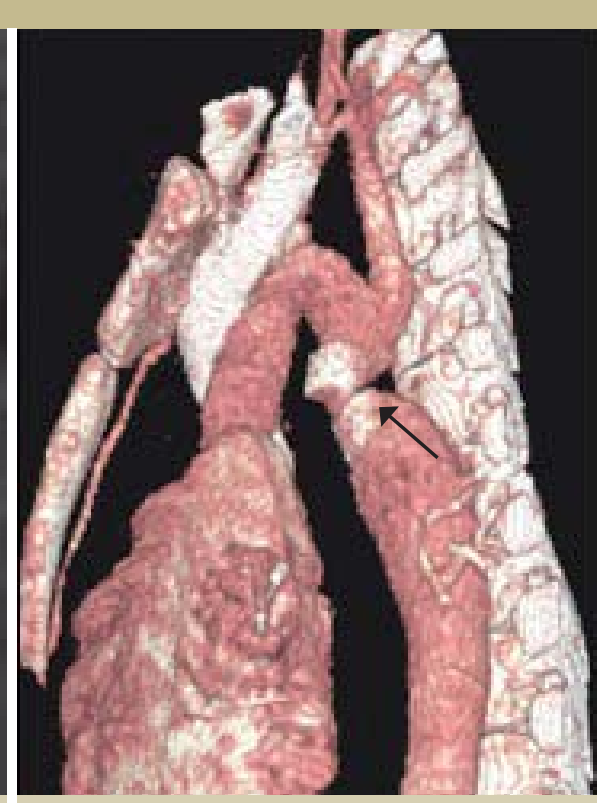

C: Volume rendered MDCT image demonstrating stent fracture at the site of the previously treated aortic coarctation. 


\section{REFERENCES:}

I. Therrien J, Dore A, Gersony W, Iserin L, Liberthson R, Meijboom F, Colman JM, Oechslin E, Taylor D, Perloff J, et al. 200 I. CCS Consensus Conference 200I update: recommendations for the management of adults with congenital heart disease. Part I. Can J Cardiol 17:940-959.

2. Fellows KE and Hubbard AM 1999. MRI: complacency or ascendancy in the evaluation of congenital heart disease. Pediatr Radiol 29:491-495.

3. Smith HJ 1999. Cardiac MR imaging. Acta Radiol 40:1-22.

4. Didier D, Ratib O, Beghetti M, Oberhaensli I and Friedli B 1999. Morphologic and functional evaluation of congenital heart disease by magnetic resonance imaging. J Magn Reson Imaging 10:639-655

5. De Roos A and Roest AA 2000. Evaluation of congenital heart disease by magnetic resonance imaging. Eur Radiol 10:2-6.

6. Higgins CB, Byrd BF, 3rd, Farmer DW, Osaki L, Silverman NH and Cheitlin MD 1984 Magnetic resonance imaging in patients with congenital heart disease. Circulation 70:851-860.

7. Shellock FG and Shellock VJ 1999. Metallic stents: evaluation of MR imaging safety. AJR Am J Roentgenol 173:543-547.

8. Razavi R, Miquel M and Baker E 2004. Diagnosis of hemi-truncus arteriosis by threedimensional magnetic resonance angiography. Circulation 109:EI5-16.

9. Bellenger NG, Marcus NJ, Rajappan K, Yacoub M, Banner NR and Pennell DJ 2002. Comparison of techniques for the measurement of left ventricular function following cardiac transplantation.J Cardiovasc Magn Reson 4:255-263.

10. Beerbaum P, Korperich H, Barth P, Esdorn H, Gieseke J and Meyer H 2001 . Noninvasive quantification of left-to-right shunt in pediatric patients: phase-contrast cine magnetic resonance imaging compared with invasive oximetry. Circulation 103:2476-2482.

II. Holmqvist C, Hochbergs P, Bjorkhem G, Brockstedt S and Laurin S 2001. Preoperative evaluation with $M R$ in tetralogy of Fallot and pulmonary atresia with ventricular septal defect. Acta Radiol 42:63-69.

12. Rebergen SA, Chin JG, Ottenkamp J, van der Wall EE and de Roos A 1993 Pulmonary regurgitation in the late postoperative follow-up of tetralogy of Fallot. Volumetric quantitation by nuclear magnetic resonance velocity mapping. Circulation 88:2257-2266

13. Taylor AM, Dymarkowski S, Hamaekers P, Razavi R, Gewillig M, Mertens L and Bogaert J 2005. MR coronary angiography and late-enhancement myocardial MR in children who underwent arterial switch surgery for transposition of great arteries. Radiology 234:542-547

14. Hoffman JI and Kaplan S 2002. The incidence of congenital heart disease. J Am Coll Cardiol 39:1890-1900

15. Lillehei CW, Varco RL, Cohen M, Warden HE, Gott VL, DeWall RA, Patton C and Moller JH 1986. The first open heart corrections of tetralogy of Fallot. A 26-3I year follow-up of 106 patients. Ann Surg 204:490-502.

16. Marshall AC, Love BA, Lang P, Jonas RA, del Nido PJ, Mayer JE and Lock JE 2003. Staged repair of tetralogy of Fallot and diminutive pulmonary arteries with a fenestrated ventricular septal defect patch. JThorac Cardiovasc Surg 126: | 427-1 433.

17. Beekman RP, Beek FJ and Meijboom EJ 1997. Usefulness of MRI for the preoperative evaluation of the pulmonary arteries in Tetralogy of Fallot. Magn Reson Imaging 15:1005-1015.

18. Nollert G, Fischlein T, Bouterwek S, Bohmer C, Dewald O, Kreuzer E, Welz A, Netz H, Klinner W and Reichart B 1997. Long-term results of total repair of tetralogy of Fallot in adulthood: 35 years follow-up in 104 patients corrected at the age of 18 or older Thorac Cardiovasc Surg 45: 178-181.

19. Nollert G, Fischlein T, Bouterwek S, Bohmer C, Klinner W and Reichart B 1997. Longterm survival in patients with repair of tetralogy of Fallot: 36-year follow-up of 490 survivors of the first year after surgical repair. J Am Coll Cardiol 30:1374- 383.
20. Bonhoeffer P, Boudjemline Y, Qureshi SA, Le Bidois J, Iserin L, Acar P, Merckx J, Kachaner J and Sidi D 2002. Percutaneous insertion of the pulmonary valve. J Am Coll Cardiol 39:1664-1669

21. Kang IS, Redington AN, Benson LN, Macgowan C, Valsangiacomo ER, Roman K, Kellenberger C] and Yoo SJ 2003. Differential regurgitation in branch pulmonary arteries after repair of tetralogy of Fallot: a phase-contrast cine magnetic resonance study Circulation 107:2938-2943.

22. Greenberg SB, Crisci KL, Koenig P, Robinson B, Anisman P and Russo P 1997. Magnetic resonance imaging compared with echocardiography in the evaluation of pulmonary artery abnormalities in children with tetralogy of Fallot following palliative and corrective surgery. Pediatr Radiol 27:932-935.

23. Vliegen HW, van Straten $A$, de Roos A, Roest AA, Schoof PH, Zwinderman AH, Ottenkamp J, van derWall EE and Hazekamp MG 2002. Magnetic resonance imaging to assess the hemodynamic effects of pulmonary valve replacement in adults late after repair of tetralogy of fallot. Circulation 106:1703-1707.

24. Helbing WA, Niezen RA, Le Cessie S, van der Geest RJ, Ottenkamp J and de Roos A 1996. Right ventricular diastolic function in children with pulmonary regurgitation after repair of tetralogy of Fallot: volumetric evaluation by magnetic resonance velocity mapping. J Am Coll Cardiol 28:1827-1835.

25. Geva T, Greil GF, Marshall AC, Landzberg M and Powell AJ 2002. Gadolinium-enhanced 3-dimensional magnetic resonance angiography of pulmonary blood supply in patients with complex pulmonary stenosis or atresia: comparison with x-ray angiography. Circulation 106:473-478.

26. Martinez JE, Mohiaddin RH, Kilner PJ, Khaw K, Rees S, Somerville J and Longmore DB 1992. Obstruction in extracardiac ventriculopulmonary conduits: value of nuclear magnetic resonance imaging with velocity mapping and Doppler echocardiography. J Am Coll Cardiol 20:338-344.

27. Haas F, Wottke M, Poppert $H$ and Meisner H 1999. Long-term survival and functional follow-up in patients after the arterial switch operation. Ann Thorac Surg 68: 1692-1697.

28. Gutberlet M, Boeckel T, Hosten N,Vogel M, Kuhne T, Oellinger H, Ehrenstein T, Venz S, Hetzer R, Bein G, et al. 2000. Arterial switch procedure for D-transposition of the great arteries: quantitative midterm evaluation of hemodynamic changes with cine MR imaging and phase-shift velocity mapping-initial experience. Radiology 21 4:467-475.

29. Hardy CE, Helton G], Kondo C, Higgins SS, Young NJ and Higgins CB 1994. Use-fulness of magnetic resonance imaging for evaluating great-vessel anatomy after arterial switch operation for D-transposition of the great arteries. Am Heart J 128:326-332.

30. Fogel MA, Hubbard A and Weinberg PM 200I. A simplified approach for assessment of intracardiac baffles and extracardiac conduits in congenital heart surgery with two- and three-dimensional magnetic resonance imaging. Am Heart J | 42: I028- 1036.

31. Sampson C, Kilner PJ, Hirsch R, Rees RS, Somerville J and Underwood SR 1994. Venoatrial pathways after the Mustard operation for transposition of the great arteries: anatomic and functional MR imaging. Radiology 193:211-217.

32. Abu-Soud HM and Hazen SL 2000. Nitric oxide is a physiological substrate for mammalian peroxidases. J Biol Chem 275:37524-37532.

33. Alam R 1997. Chemokines in allergic inflammation. J Allergy Clin Immunol 99:273-277. 34. Gomes AS, Lois, JF, George B, Alpan G and Williams RG 1987. Congenital abnormalities of the aortic arch: MR imaging. Radiology | 65:69|-695.

35. Von Schulthess GK, Higashino SM, Higgins SS, Didier D, Fisher MR and Higgins CB 1986. Coarctation of the aorta: MR imaging. Radiology 158:469-474.

36. Holmqvist C, Stahlberg F, Hanseus K, Hochbergs P, Sandstrom S, Larsson EM and Laurin S 2002. Collateral flow in coarctation of the aorta with magnetic resonance velocity mapping: correlation to morphological imaging of collateral vessels. J Magn Reson Imaging 15:39-46. 
37. Debatin JF and Hany TF 1998. MR-based assessment of vascular morphology and function. Eur Radiol 8:528-539.

38. Mohiaddin RH, Kilner PJ, Rees S and Longmore DB 1993. Magnetic resonance volume flow and jet velocity mapping in aortic coarctation. J Am Coll Cardiol 22:1515-1521.

39. Fattori R and Nienaber CA 1999. MRI of acute and chronic aortic pathology: preoperative and postoperative evaluation. J Magn Reson Imaging 10:741-750.

40. Bogaert J, Gewillig M, Rademakers F, Bosmans H,Verschakelen J, Daenen W and Baert AL 1995. Transverse arch hypoplasia predisposes to aneurysm formation at the repair site after patch angioplasty for coarctation of the aorta. J Am Coll Cardiol 26:52I-527.

4I. Kaemmerer H, Theissen P, Konig U, Sechtem U and de Vivie ER 1993. Follow-up using magnetic resonance imaging in adult patients after surgery for aortic coarctation. Thorac Cardiovasc Surg 41:107-111.

42. Higgins $C B$ and Sakuma $H$ 1996. Heart disease: functional evaluation with MR imaging Radiology 199:307-315.

43. Kersting-Sommerhoff BA, Sechtem UP, Fisher MR and Higgins CB 1987. MR imaging of congenital anomalies of the aortic arch. AJR Am J Roentgenol 149:9-13.

44. Roman MJ, Devereux RB, Niles NW, Hochreiter C, Kligfield P, Sato N, Spitzer MC and Borer JS 1987. Aortic root dilatation as a cause of isolated, severe aortic regurgitation. Prevalence, clinical and echocardiographic patterns, and relation to left ventricular hypertrophy and function. Ann Intern Med 106:800-807.

45. Mohiaddin RH, Schoser K, Amanuma M, Burman ED and Longmore DB 1990. MR imaging of age-related dimensional changes of thoracic aorta. J Comput Assist Tomogr 14:748-752.

46. Hamdan MA, Maheshwari S, Fahey JT and Hellenbrand WE 200 I. Endovascular stents for coarctation of the aorta: initial results and intermediate-term follow-up. J Am Coll Cardiol 38:1518-1523.

47. Dake MD, Miller DC, Mitchell RS, Semba CP, Moore KA and Sakai T 1998. The "first generation" of endovascular stent-grafts for patients with aneurysms of the descending thoracic aorta. JThorac Cardiovasc Surg I I 6:689-703; discussion 703-684.

48. Fattori R, Napoli G, Lovato L, Grazia C, Piva T, Rocchi G, Angeli E, Di Bartolomeo R and Gavelli G 2003. Descending thoracic aortic diseases: stent-graft repair. Radiology 229:176-183.

49. Fischer G, Kramer HH, Stieh J, Harding P and Jung O. 1999. Transcatheter closure of secundum atrial septal defects with the new self-centering Amplatzer Septal Occluder Eur Heart J 20:541 -549.

50. Fischer G, Stieh J, Uebing A, Hoffmann U, Morf G and Kramer HH 2003. Experience with transcatheter closure of secundum atrial septal defects using the Amplatzer septal occluder: a single centre study in 236 consecutive patients. Heart 89:199-204.

5I. Durongpisitkul K, Tang NL, Soongswang J, Laohaprasitiporn D and Nanal A 2004 Predictors of successful transcatheter closure of atrial septal defect by cardiac magnetic resonance imaging. Pediatr Cardiol 25:124-130.

52. Taylor AM, Stables RH, Poole-Wilson PA and Pennell DJ 1999. Definitive clinical assessment of atrial septal defect by magnetic resonance imaging. J Cardiovasc Magn Reson 1:43-47.

53. Lange A, Walayat M, Turnbull CM, Palka P, Mankad P, Sutherland GR and Godman M 1997. Assessment of atrial septal defect morphology by transthoracic three dimensional echocardiography using standard grey scale and Doppler myocardial imaging techniques: comparison with magnetic resonance imaging and intraoperative findings. Heart 78:382-389

54. Langley SM, Winlaw DS, Stumper O, Dhillon R, De Giovanni JV, Wright JG, Miller P, Sethia B, Barron DJ and Brawn WJ 2003. Midterm results after restoration of the morphologically left ventricle to the systemic circulation in patients with congenitally corrected transposition of the great arteries. JThorac Cardiovasc Surg |25: |229- |241.
55. Miquel ME, Hill DL, Baker El. Qureshi SA, Simon RD, Keevil SF and Razavi RS 2003. Three- and four-dimensional reconstruction of intra-cardiac anatomy from twodimensional magnetic resonance images. Int J Cardiovasc Imaging 19:239-254; discussion 255-236.

56. Razavi RS, Hill DL, Muthurangu V, Miquel ME, Taylor AM, Kozerke S and Baker E) 2003. Three-dimensional magnetic resonance imaging of congenital cardiac anomalies. Cardiol Young | 3:46|-465.

57. Puvaneswary M, Leitch J and Chard RB 2003. MRI of partial anomalous pulmonary venous return (scimitar syndrome). Australas Radiol 47:92-93.

58. Fogel MA and Rychik J 1998. Right ventricular function in congenital heart disease: pressure and volume overload lesions. Prog Cardiovasc Dis 40:343-356.

59. Muthurangu V, Taylor A, Andriantsimiavona R, Hegde S, Miquel ME, Tulloh R, Baker E, Hill DL and Razavi RS 2004. Novel method of quantifying pulmonary vascular resistance by use of simultaneous invasive pressure monitoring and phase-contrast magnetic resonance flow. Circulation I 1 0:826-834

60. Simpson IA, Valdes-Cruz LM, Berthoty DP, Powell JB, Hesselink JR, Chung KJ and Sahn DJ 1993. Cine magnetic resonance imaging and color Doppler flow mapping in infants and children with pulmonary artery bands. Am I Cardiol 71:1419-1426.

6I. Taylor AJ, Byers JP, Cheitlin MD and Virmani R 1997. Anomalous right or left coronary artery from the contralateral coronary sinus: "high-risk" abnormalities in the initia coronary artery course and heterogeneous clinical outcomes. Am Heart J 133:428-435.

62. Taylor AM, Thorne SA, Rubens MB, Jhooti P, Keegan J, Gatehouse PD, Wiesmann F Grothues F, Somerville J and Pennell DJ 2000. Coronary artery imaging in grown up congenital heart disease: complementary role of magnetic resonance and $x$-ray coronary angiography. Circulation 101:1670-1678,

63. Giorgi B, Dymarkowski S, Rademakers FE, Lebrun F and Bogaert I 2002. Single coronary artery as cause of acute myocardial infarction in a 12-year-old girl: a comprehensive approach with MR imaging. AIR Am J Roentgenol 179:1535-1537.

64. Westra SJ, Hill JA, Alejos JC, Galindo A, Boechat MI and Laks H 1999. Three-dimensional helical CT of pulmonary arteries in infants and children with congenital heart disease. AJR Am J Roentgenol 173:109-1 I5.

65. Kawano T, Ishii M, Takagi J, Maeno Y, Eto G, Sugahara Y, Toshima T, Yasunaga $H$ Kawara T, Todo K, et al. 2000. Three-dimensional helical computed tomographic angiography in neonates and infants with complex congenital heart disease. Am Heart J 139:654-660

66. Lawler LP and Fishman EK 200I. Multi-detector row CT of thoracic disease with emphasis on 3D volume rendering and CT angiography. Radiographics 21:1257-1273.

67. Siegel MJ 2003. Multiplanar and three-dimensional multi-detector row CT of thoracic vessels and airways in the pediatric population. Radiology 229:641 -650.

68. Allen JK, Hensley WJ, Nicholls AV and Whitfield JB 1979. An enzymic and centrifugal method for estimating high-density lipoprotein cholesterol. Clin Chem 25:325-327.

69. Ameli S, Hultgardh-Nilsson A, Cercek B, Shah PK, Forrester JS, Ageland H and Nilsson 1994. Recombinant apolipoprotein A-I Milano reduces intimal thickening after balloon injury in hypercholesterolemic rabbits. Circulation 90:1935-1941.

70. Achenbach S, Ropers D, Kuettner A, Flohr T, Ohnesorge B, Bruder H, Theessen H, Karakaya M, Daniel WG, Bautz W, et al. 2006. Contrast-enhanced coronary artery visualization by dual-source computed tomography-initial experience. Eur J Radiol 57:331-335

71. Achenbach S, Ropers D, Pohle FK, Raaz D, von Erffa J, Yilmaz A, Muschiol G and Daniel WG 2005. Detection of coronary artery stenoses using multi-detector CT with $16 \times 0.75$ collimation and 375 ms rotation. Eur Heart J 26:1978-1986.

72. Mollet NR, Cademartiri F and de Feyter PJ 2005. Non-invasive multislice CT coronary imaging. Heart 91:401-407. 